\title{
$1 \leftrightarrow 2$ processes of a sterile neutrino around the electroweak scale in a thermal plasma
}

\author{
Xue-Min Jiang, Yi-Lei Tang, ${ }^{*}$ Zhao-Huan Yu®, and Hong-Hao Zhang ${ }^{\dagger}$ \\ School of Physics, Sun Yat-Sen University, Guangzhou 510275, China
}

(Received 14 December 2020; revised 22 March 2021; accepted 2 April 2021; published 6 May 2021)

In this paper, we apply the Goldstone equivalence gauge to calculate the $1 \leftrightarrow 2$ processes of a sterile neutrino in a thermal plasma below the standard model (SM) critical temperature $T_{c} \approx 160 \mathrm{GeV}$. The sterile neutrino's mass is around the electroweak scale, $50 \mathrm{GeV} \leq m_{N} \leq 200 \mathrm{GeV}$, and the acquired thermal averaged effective width $\bar{\Gamma}_{\text {tot }}$ does not depend on the gauge selection and is continuous around the crossover. We also apply our results to perform a preliminary calculation of leptogenesis.

DOI: 10.1103/PhysRevD.103.095003

\section{INTRODUCTION}

Sterile neutrinos interacting with the plasma background of the early Universe can become a potential solution to some cosmological particle physics problems. A prominent example is leptogenesis [1]. The $C P$-violation effects of sterile neutrino interactions with light leptons give rise to the lepton-number asymmetry in the plasma, and the baryon-number asymmetry accordingly appears through the sphaleron effects (for some early works, see Refs. [2-6], and see Refs. [7-10] for reviews). The sterile neutrino can also become a portal to dark matter. Being a variation of a secluded dark matter model, a "sterileneutrino-philic dark matter" model [11-18] gives a different relic density result when compared with the standard weakly interacting massive particle (WIMP) models [19]. In Ref. [20], we also studied a feebly interacting massive particle (FIMP) [21] version of such a model. Sometimes, sterile neutrinos themselves can also become the dark matter candidate. In all these examples, a reliable calculation of the sterile neutrino's interaction with the thermal plasma is crucial for precise predictions of the related physical observables compared with the experimental data.

When $m_{N} \gg T_{c} \simeq 160 \mathrm{GeV}$, where $m_{N}$ is the sterile neutrino mass and $T_{c}$ is the electroweak crossover temperature [22], there are plenty of reliable discussions in the literature to calculate the sterile neutrino's production [23-31]. Since the crucial temperature $T \sim m_{N}$ is well above the crossover temperature, only the Higgs doublet

\footnotetext{
*tangylei@mail.sysu.edu.cn

zhh98@mail.sysu.edu.cn
}

Published by the American Physical Society under the terms of the Creative Commons Attribution 4.0 International license. Further distribution of this work must maintain attribution to the author(s) and the published article's title, journal citation, and DOI. Funded by SCOAP . and the active leptons participate in the $1 \leftrightarrow 2$ processes. The Higgs components receive a universal thermal mass correction, which is easily calculated. For lighter sterile neutrinos, successful leptogenesis can also be acquired through the resonant effects [32-38]. When $m_{N} \ll T_{c}$, at $T \sim m_{N} \ll T_{c}$, the thermal mass terms can be safely neglected, since the vacuum expectation value (VEV) of the Higgs boson becomes fairly close to the zero-temperature value $\sim 246 \mathrm{GeV}$, and the boson's behaviors are similar to those in the zero-temperature situation [39].

In the literature, there seems to be a gap where $m_{N} \sim T_{c}$. In this range, the calculation is plagued by the intricate thermal corrections to the gauge and Higgs sectors. In Ref. [40], the authors estimated the $\mathrm{U}(1)_{Y} \times \mathrm{SU}(2)_{L}$ gauge boson contributions by replacing them with the Goldstone degrees of freedom artificially assigned with the similar mass of the Higgs boson. We also applied this method in the corresponding calculations of our papers $[12,20]$. Such an ansatz might be inspired by the famous "Goldstone equivalence theorem" at zero temperature, which requires more investigation in the thermal plasma case. A safe procedure is to return to the original form of the finitetemperature propagators to integrate all the branch cuts and poles that may appear, as described in Ref. [41-44]. However, the procedures to follow there are formidable, and the relationship between the Goldstone and gauge boson becomes more obscure. Another problem is that the invariant squared mass of the sterile neutrino, which is denoted by $\mathcal{K}^{2}$ in Refs. [41-44], had been neglected around $T_{c}$ there, so their method is not suitable to our $\mathcal{K}^{2}=$ $m_{N}^{2} \sim T_{c}^{2}$ range of interest.

In Ref. [45], we proposed a method to decompose the massive gauge boson propagators in the thermal plasma with the Goldstone equivalence gauge. Poles indicating the "transverse" and "longitudinal" degrees of freedom arise as usual, and a branch cut which closely resembles two 
massless poles was identified as the Goldstone boson's fragment. When $T>T_{c}$, such a branch cut fragments into two actual poles corresponding to the Goldstone boson particles, and when $T=0$, this branch cut completely disappears. In the finite-temperature case, the longitudinal polarization is also some intermediate state between the so-called "plasmon" and the Goldstone equivalent state. We made an analogy that the longitudinal polarization will "spew out" a fraction of the Goldstone boson in the finitetemperature environment. This helps us to include all the contributions from the transverse, longitudinal, Higgs, and Goldstone degrees of freedom correctly, and it helps us to clarify the relationship between the Goldstone and the gauge bosons in the plasma. We note that Ref. [45] also provides a typical $R_{\xi}$ method, which is equivalent to our Goldstone equivalence gauge results if the gauge vertex hard thermal loop (HTL) corrections denoted in Eq. (A4) are included. We will consider these corrections in our Eqs. (17) and (32) for the gauge independent results.

In this paper, with the method we have developed in Ref. [45], we will calculate the sterile neutrino $1 \leftrightarrow 2$ processes near the electroweak crossover temperature $m_{N} \sim T \sim T_{c}$. We will also roughly discuss the leptogenesis induced by these processes. A complete calculation of the sterile neutrino's interaction in the early Universe should also include the more complicated $2 \leftrightarrow 2$ scattering processes. In many cases, when $T \gg m_{N}$, and when the $l-H-N$ Yukawa couplings are sufficiently large, $y_{N} \gtrsim 10^{-8}$, the thermal equilibrium of the sterile neutrino does not require a detailed calculation. When the temperature drops down to the $T \sim m_{N}$ scale, the out-of-equilibrium effects start to arise, and these $2 \leftrightarrow 2$ processes are usually suppressed rapidly due to an additional number-density factor compared with the $1 \leftrightarrow 2$ processes. With these considerations, we leave the $2 \leftrightarrow 2$ processes to future study and do not consider their contributions at this stage. For brevity and simplicity, we also do not consider the contributions resumming the interchange and emission of the soft bosons [46-48] (sometimes called the LPM resummation) in this paper.

We enumerate the channels and list the basic formulas in Sec. II. Details on phase space and thermal integrals are presented in Sec. III. Numerical results and a preliminary calculation of leptogenesis are displayed in Sec. IV. We summarize this paper in Sec. V.

\section{BASIC CONCEPTS AND CHANNEL ENUMERATION}

The Lagrangian for sterile neutrinos is the standard one:

$$
\mathcal{L} \supset \mathcal{L}_{\mathrm{SM}}+\mathcal{L}_{\mathrm{Nkin}}+\mathcal{L}_{\mathrm{N} \text { mass }}-\sqrt{2} y_{N i j} H \bar{l}_{i} N_{j}+\text { H.c. },
$$

where $H$ is the Higgs doublet; $L_{i}$, with $i=1,2,3$, are the lepton doublets of three generations; and $N_{j}$ are the sterile neutrinos. $N_{j}$ can be either Majorana or (pseudo-)Dirac spinors, and the corresponding kinematical and mass terms $\mathcal{L}_{\mathrm{N} \text { kin }}+\mathcal{L}_{\mathrm{N} \text { mass }}$ differ by a factor of $\frac{1}{2}$. For simplicity, here we only study the one Dirac sterile neutrino case. The interaction only involves one massless lepton. A general situation can be inferred from our results by simply multiplying some factors. Therefore, the Lagrangian we are relying on is given by

$$
\mathcal{L} \supset \mathcal{L}_{\mathrm{SM}}+i \bar{N} \not \partial N-m_{N} \bar{N} N-\sqrt{2} y_{N} H \bar{l} N+\text { H.c. },
$$

where $m_{N}$ is the mass of the sterile neutrino.

Above the standard model (SM) critical temperature of the crossover $T>T_{c} \approx 160 \mathrm{GeV}$, the $1 \leftrightarrow 2$ processes of the sterile neutrino have nothing to do with the $W / Z$ boson. Only the Higgs doublets including the Goldstone components participate in the couplings. The whole process is quite standard: the thermal effects correct the effective Higgs mass term

$$
\delta m_{H, \text { thermal }}^{2}=\left(g_{1}^{2}+3 g_{2}^{2}+4 y_{t}^{2}+8 \lambda\right) \frac{T^{2}}{16},
$$

where $g_{1}, g_{2}$ are the electroweak gauge coupling constants; $y_{t}$ is the top Yukawa coupling constant; and $\lambda$ is the fourHiggs coupling constant. Leptons also receive thermal mass corrections. In the thermal plasma, each pole in the leptonic propagators is split into two objects, called a "particle" and a "hole." In Ref. [23], both of these two objects are combined into one single particle with the universal thermal mass correction to estimate the phase space. In this paper, we abandon this approximation, and we earnestly sum over each contribution from these two degrees of freedom.

Below the critical temperature $T<T_{c}$, the vacuum expectation value (VEV) is estimated to be $v(T)=$ $v_{0} \sqrt{1-\frac{T^{2}}{T_{c}^{2}}}$, where $v_{0}=246 \mathrm{GeV}$. This opens the sterile neutrino's oscillation into a highly off-shell active neutrino, and then it decays into a $W / Z$ gauge boson plus a charged lepton/active neutrino. An on-shell $W / Z$ boson can also decay into a pair of leptons, and the active neutrino product can also oscillate into a sterile neutrino through the VEV.

The dispersion relations (or the "on-shell" equation) of the $W / Z$ bosons below the critical temperature are complicated. Together with the dispersion relations of the leptons and the conservation of energy and momentum equations, we have four equations to solve the phase space. Three of them are transcendental equations. Later, we are going to describe the details to solve them.

In this paper, we rely on the Goldstone equivalent gauge [49] to calculate the sterile neutrino's productions in the thermal plasma [45] below the critical temperature $T_{c}$. Within this framework, each Goldstone degree of freedom is attributed to two parts: one is hidden inside the extended 
polarization vector of a longitudinal vector boson, while the other behaves like a massless particle during the calculations and is regarded independently as a Goldstone boson's fraction. We enumerate and include all of the gauge polarizations and the Goldstone boson fraction's contributions. In the Appendix, we will also show the equivalence between this gauge and the usually familiar $R_{\xi}$ gauge.

In the following subsections, we will describe the details for each channel. Before starting them, we also note that we ignore some of the subdominant tachyonic branch cuts in the bosonic propagators, as illustrated in our Ref. [45]. As in Ref. [23], the subdominant branch cuts in the leptonic propagators are also neglected.

\section{A. $W$ channels}

The Feynmann diagram of a sterile neutrino $N$ decaying into a $W^{+}$boson and a charged lepton $l^{-}$is illustrated in Fig. 1. Since we are discussing a Dirac $N$, it is possible to invert the arrows there to reformulate it into an $\bar{N}$-decay diagram. We neglect the antisterile neutrino's decay in our paper, since the results are completely symmetric, by neglecting the $C P$ effects. The momentum flows are also defined in Fig. 1 and are defined relative to the plasma background reference-i.e., the plasma's fourvector velocity

$$
u^{\mu}=(1,0,0,0)
$$

When, e.g., $p_{1}^{0}<0$, the same diagram can also be interpreted as a charged lepton's fusion with the sterile neutrino to generate a $W^{+}$boson, which is the dual process of a $W^{+}$ decaying into a $N, l^{+}$pair. This is the "inverse decay" process of a $W^{+}$boson, and we denote it with "ID" for abbreviation later. The thermal equilibrium condition guarantees the equality of the results from both aspects of the "decay" and "inverse decay" processes of a $W$ boson. Therefore, Fig. 1 can summarize all the possible $1 \leftrightarrow 2$ processes of an (anti)sterile neutrino.

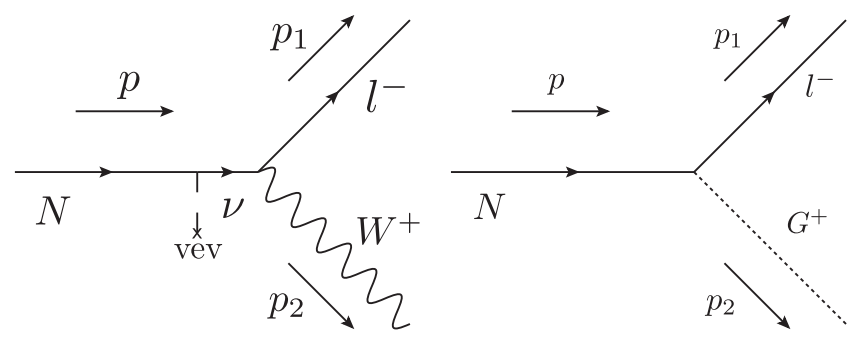

FIG. 1. $\quad N \rightarrow W^{+} l^{-} 1 \leftrightarrow 2$ channel. Since we have applied the Goldstone equivalence gauge, the Goldstone contribution is explicitly contained in the polarization vector, so we also need to calculate the Goldstone part of the diagrams.
The dispersion relation of a $W$ boson is given by

$$
F_{W,(L, T)}\left(p_{2}\right)=p_{2}^{2}-\left[m_{W}(T)\right]^{2}-\Pi_{L, T}^{W}\left(p_{2}\right)=0,
$$

for transverse and longitudinal polarizations, respectively, where

$$
\begin{aligned}
& \Pi_{L}^{W}\left(p_{2}\right)=-\frac{2 m_{E 2}^{2} p_{2}^{2}}{\vec{p}_{2}^{2}}\left(1-\frac{p_{2}^{0}}{\left|\vec{p}_{2}\right|} Q_{0}\left(\frac{p_{2}^{0}}{\left|\vec{p}_{2}\right|}\right)\right), \\
& \Pi_{T}^{W}\left(p_{2}\right)=\frac{1}{2}\left(2 m_{E 2}^{2}-\Pi_{L}^{W}\left(p_{2}\right)\right),
\end{aligned}
$$

and

$$
Q_{0}(x)=\frac{1}{2} \ln \frac{x+1}{x-1} .
$$

The VEV-dependent $W$-boson mass is given by

$$
m_{W}(T)=\frac{g_{2} v(T)}{2},
$$

where $g_{2}$ is the weak coupling constant, and the Debye thermal mass $m_{E 2}$ takes the form

$$
m_{E 2}^{2}=\frac{11}{6} g_{2}^{2} T^{2}
$$

Ignoring the lepton's VEV-dependent mass, since it is much smaller than the thermal mass term, the thermalcorrected dispersion relation of the active lepton is given (see p. 140 in Ref. [50]) by

$$
F_{l}\left(p_{1}\right)=\left[\Delta_{+}\left(p_{1}\right) \Delta_{-}\left(p_{2}\right)\right]^{-1}=0
$$

where

$$
\begin{aligned}
\Delta_{ \pm}\left(p_{1}\right)= & \left(p_{1}^{0} \mp\left|\vec{p}_{1}\right|\right. \\
& \left.-\frac{m_{f}^{2}}{2\left|\vec{p}_{1}\right|}\left[\left(1 \mp \frac{p_{1}^{0}}{\left|\vec{p}_{1}\right|}\right) \ln \frac{p_{1}^{0}+\left|\vec{p}_{1}\right|}{p_{1}^{0}-\left|\vec{p}_{1}\right|} \pm 2\right]\right)^{-1} .
\end{aligned}
$$

Here,

$$
m_{f}^{2}=\frac{g_{1}^{2}+3 g_{2}^{2}}{32} T^{2}
$$

Generally, there are four solutions to Eq. (10). When $p_{1}^{2}>m_{f}^{2}$, this means a "particle" for $p_{1}^{0}>0$ and an "antiparticle" for $p_{1}^{0}<0$. When $p_{1}^{2}<m_{f}^{2}$, this indicates a "hole" for $p_{1}^{0}>0$ and an "antihole" for $p_{1}^{0}<0$.

The energy and momentum conservation laws are given by 


$$
\begin{gathered}
p^{0}=p_{1}^{0}+p_{2}^{0}, \\
\vec{p}_{2}^{2}=\vec{p}^{2}+\vec{p}_{1}^{2}-2\left|\vec{p} \| \vec{p}_{1}\right| \cos \theta_{p},
\end{gathered}
$$

where $\theta_{p}$ is the angle between $\vec{p}$ and $\vec{p}_{1}$. The subscript " $p$ " denotes the plasma, which means that this is the angle measured in the plasma rest frame. Given the sterile neutrino's energy and momentum $p^{0}, \vec{p}$, and fixing the $\theta_{p}$, there are four unknown parameters $-p_{1}^{0}, p_{2}^{0},\left|\vec{p}_{1}\right|$, $\left|\vec{p}_{2}\right|$-in just four equations: (5), (10), (13), and (14). Solving these equations might give a set of solutions. If $p_{1}^{0}$ or $p_{2}^{0}$ is smaller than zero, it means that a lepton or a $W$ boson becomes an initial-state particle. We need to find all of the solutions to sum over all their contributions to the "interaction rate" $\gamma_{N}$.

With the acquired $p_{1}$ and $p_{2}$, we can then calculate the amplitude. In the Goldstone equivalence gauge, the "polarization vector" of a gauge boson is extended to a five-component vector $\epsilon_{ \pm, L \text { in }}^{W n}\left(p_{2}\right)=\epsilon_{ \pm, L \text { out }}^{W n *}\left(p_{2}\right), n=\mu, 4$, to include the Goldstone component (where $n=4$ denotes the Goldstone component). When contracting the indices, the metric tensor $\left[g^{\mu \nu}\right]=\operatorname{diag}[1,-1,-1,-1]$ is also extended to $\left[g^{m n}\right]=\operatorname{diag}[1,-1,-1,-1,-1]$. The transverse polarization is the same as in the $R_{\xi}$ gauge with $\epsilon_{ \pm}^{W 4}\left(p_{2}\right)=\epsilon_{ \pm}^{W 0}\left(p_{2}\right)=0$, and $\epsilon_{ \pm}^{W i}\left(p_{2}\right) p_{2 i}=0$. The longitudinal polarization $\epsilon_{\mathrm{Lin}}^{W n *}\left(p_{2}\right)=\epsilon_{\mathrm{Lout}}^{W n}\left(p_{2}\right)$ is given by

$$
\epsilon_{\text {Lout }}^{W}\left(p_{2}\right)=\left(\begin{array}{c}
-\frac{\sqrt{p_{2}^{2}}}{n_{2} \cdot p_{2}} n_{2}^{\mu} \\
-i \frac{m_{W}(T)}{\sqrt{p_{2}^{2}}}
\end{array}\right),
$$

where $n_{2}^{\mu}=\left(1,-\frac{\vec{p}_{2}}{\left|\vec{p}_{2}\right|}\right)$ for the convention of $\left(k^{\mu}\right)=\left(k^{0}, \vec{k}\right)$ for any four-dimensional momentum $k$.

For the lepton spinors, we need to define

$$
\tilde{p}_{1}=p_{1}^{0}\left(1, \pm \frac{\vec{p}_{1}}{\left|\vec{p}_{1}\right|}\right)
$$

where for a "particle"-i.e., $p_{1}^{2}>m_{f}^{2}$-the "+" sign is adopted, and for a "hole"-i.e., $p_{1}^{2}<m_{f}^{2}$ - the " -" sign is adopted. When $p_{1}^{0}>0$, a lepton (either a "particle" or a "hole") is created, and a $\bar{u}^{s}\left(\tilde{p}_{1}\right)$ appears in the amplitude. When $p_{1}^{0}<0$, an antilepton (either an "antiparticle" or an "antihole") is destroyed, and a $\bar{v}^{s}\left(-\tilde{p}_{1}\right)$ appears in the amplitude.

The amplitude of the gauge component, as denoted in the left panel of Fig. 1, then becomes

$i \mathcal{M}_{W}^{\mu}=-y_{N} v(T) \frac{g_{2}}{\sqrt{2}} \bar{u}^{s}\left(\tilde{p}_{1}\right)\left[\gamma^{\mu}+\Gamma^{\mu}\left(p, p_{1}\right)\right] P_{L} \frac{i}{\not p_{l T}} u^{r}(p)$, when $p_{1}^{0}>0$ for the decay channel. $\Gamma^{\mu}\left(p, p_{1}\right)$ is the HTL correction on the gauge vertex introduced for a gaugeinvariant result. Its definition is given in Eq. (A4), followed by the detailed evaluation processes in the Appendix. If $p_{1}^{0}<0$, we only need to change $\bar{u}^{s}\left(\tilde{p}_{1}\right)$ into $\bar{v}^{s}\left(-\tilde{p}_{1}\right)$ for the $W$ boson's inverse-decay channel. The Goldstone component of the amplitude, as denoted in the right panel of Fig. 1, is written to be

$$
i \mathcal{M}_{W}^{4}=-\sqrt{2} y_{N} \bar{u}^{s}\left(\tilde{p}_{1}\right) P_{R} u^{r}(p) .
$$

Again, when $p_{1}^{0}<0, \bar{u}\left(\tilde{p}_{1}\right)$ needs to be replaced with $\bar{v}\left(-\tilde{p}_{1}\right)$. In the above equations, $P_{L, R}=\frac{1 \mp \gamma^{5}}{2}$, and the definition of $p_{l T}$ is

$$
\begin{aligned}
p_{l T} & =\left(p_{l T}^{0}, \vec{p}_{l T}\right) \\
& =\left(\left(1-\frac{m_{f}^{2} L}{p^{0}}\right) p^{0},\left(1+\frac{m_{f}^{2}\left(1-p^{0} L\right)}{\vec{p}^{2}}\right) \vec{p}\right),
\end{aligned}
$$

where

$$
L=\frac{1}{2|\vec{p}|} \ln \frac{p^{0}+|\vec{p}|}{p^{0}-|\vec{p}|} .
$$

The complete amplitude should take the form

$$
\epsilon_{(t) n}^{W}\left(p_{2}\right)\left(i \mathcal{M}_{W}^{n}\right),
$$

where $n=0,1,2,3,4, t= \pm$, Lout. The squared amplitude should also take the statistic factor and the "renormalization constant." The complete result is

$$
\begin{aligned}
A_{W, t}= & \sum_{r, s=1,2} \mathcal{M}_{W}^{n} \mathcal{M}_{W}^{* m} \epsilon_{t n}^{W} \epsilon_{t m}^{W *} f_{F}\left(\frac{p_{1}^{0}}{T}\right) \\
& \times f_{B}\left(\frac{p_{2}^{0}}{T}\right) Z_{l}\left(p_{1}\right) Z_{W t}\left(p_{2}\right),
\end{aligned}
$$

where $t= \pm$, Lout indices are not summed by the Einstein sum rule, and

$$
\begin{aligned}
& f_{F}(x)=\frac{e^{x}}{e^{x}+1}, \\
& f_{B}(x)=\left|\frac{e^{x}}{e^{x}-1}\right|,
\end{aligned}
$$

and the "renormalization factors" are

$$
\begin{gathered}
Z_{W(T, L)}\left(p_{2}\right)=\frac{2 p_{2}^{0}}{2 p_{2}^{0}-\frac{\partial \Pi_{T . L}^{W}\left(p_{2}\right)}{\partial p_{2}^{0}},} \\
Z_{l}\left(p_{1}\right)=\frac{\left(p_{1}^{0}\right)^{2}-\vec{p}_{1}^{2}}{2 m_{f}^{2}} .
\end{gathered}
$$




\section{B. $Z / \gamma$ channels}

Since $W$ and $B$ bosons receive different thermal corrections, this disturbs the mixing angle for the "on-shell" $Z / \gamma$ bosons. The mixing angles of the on-shell $Z / \gamma$ bosons depend on their energy and momentum, so it is difficult to identify which is the $Z$ or $\gamma$ degree of freedom. The corresponding diagram including both $Z / \gamma$ and the neutral Goldstone contributions are presented in Fig. 2.

The VEV-dependent mass matrix for the $B / W^{3}$ field, or $Z / \gamma$ particle, is as usual:

$$
m_{Z / \gamma}^{2}(T)=\frac{(v(T))^{2}}{4}\left(\begin{array}{cc}
g_{1}^{2} & -g_{1} g_{2} \\
-g_{1} g_{2} & g_{2}^{2}
\end{array}\right) .
$$

Thermal effects correct the $B$ and $W^{3}$ mass terms, respectively, and therefore the thermal mass matrix is given by

$$
\Pi_{T, L}^{Z / \gamma}\left(p_{2}\right)=\left(\begin{array}{cc}
\Pi_{T, L}^{B}\left(p_{2}\right) & 0 \\
0 & \Pi_{T, L}^{W}\left(p_{2}\right)
\end{array}\right),
$$

where $\Pi_{T, L}^{W}\left(p_{2}\right)$ has already been given by Eq. (6). $\Pi_{T, L}^{B}$ changes the $m_{E 2}$ in Eq. (6) into $m_{E 1}$,

$$
m_{E 1}^{2}=\frac{11}{6} g_{1}^{2} T^{2} .
$$

The dispersion rate of this mixed $Z / \gamma$ is given by the "secular equation,"

$F_{Z / \gamma,(T, L)}\left(p_{2}\right)=\operatorname{det}\left(p_{2}^{2} I_{2 \times 2}-m_{Z / \gamma}^{2}(T)-\Pi_{T, L}^{Z / \gamma}\left(p_{2}\right)\right)=0$,

for a transverse/longitudinal $Z / \gamma$ vector boson. $I_{2 \times 2}$ is the $2 \times 2$ identity matrix. For a given $p_{2}$ as a solution of Eq. (30), the matrix $p_{2}^{2} I_{2 \times 2}-m_{Z / \gamma}^{2}(T)-\Pi_{T, L}^{Z / \gamma}\left(p_{2}\right)$ has a zero eigenvalue, and the corresponding eigenvector is denoted by $x=\left(\begin{array}{l}x_{1} \\ x_{2}\end{array}\right)$, where $x_{1}^{2}+x_{2}^{2}=1$. In the zerotemperature case, $x_{1}^{Z}=-\sin \theta_{W}, x_{2}^{Z}=\cos \theta_{W}$ for the $Z$ boson, and $x_{1}^{\gamma}=\cos \theta_{W}, x_{2}^{\gamma}=\sin \theta_{W}$ for the photon, where $\theta_{W}$ is the Weinberg angle. Since the neutrino does not interact with a pure photon, we can calculate the inner product $x \cdot x^{Z}=-x_{1} \sin \theta_{W}+x_{2} \cos \theta_{W}$ to extract the $Z$ part of the "on-shell" mixed boson to calculate its interactions with the leptons. The dispersion relation of a lepton and the energy-momentum conservation law is exactly the same as with Eqs. (10), (13), and (14) in Sec. II A. Solving these equations with Eq. (30), we then acquire all the "on-shell" $p_{1}$ and $p_{2}$.

The transverse polarization vectors of a $Z / \gamma$ boson $\epsilon_{ \pm}^{Z / \gamma n}$ are the same as those of the $W$ boson $\epsilon_{ \pm}^{W n}$ to satisfy $p_{2 \mu} \epsilon_{ \pm}^{Z / \gamma \nu}=0, \epsilon_{ \pm}^{Z / \gamma 4}=0$, and $p_{2 i} \epsilon_{ \pm}^{Z / \gamma i}=0$. The longitudinal polarization vector is given by

$$
\begin{aligned}
\epsilon_{L \text { in }}^{Z / \gamma *}\left(p_{2}\right) & =\epsilon_{L \text { out }}^{Z / \gamma}\left(p_{2}\right) \\
& =\left(\begin{array}{c}
-\frac{\sqrt{p_{2}^{2}}}{n_{2} \cdot p_{2}} n_{2}^{\mu} \\
-i \frac{m_{Z}(T)}{\sqrt{p_{2}^{2}}}\left(-x_{1} \sin \theta_{W}+x_{2} \cos \theta_{W}\right)
\end{array}\right) .
\end{aligned}
$$

Compared with Eq. (15), the extra $\left(-x_{1} \sin \theta_{W}+x_{2} \cos \theta_{W}\right)$ factor in the Goldstone component indicates that only the $Z$ component of the vector boson has "eaten" some Goldstone boson; the photon part of this vector boson has not devoured any Goldstone boson's fraction.

Then, we are ready to write the amplitudes:

$$
\begin{aligned}
i \mathcal{M}_{Z / \gamma}^{\mu}= & -y_{N} v(T) \frac{g_{2}}{2 \cos \theta_{W}} \bar{u}^{s}\left(\tilde{p}_{1}\right)\left[\gamma^{\mu}+\Gamma^{\mu}\left(p, p_{1}\right)\right] P_{L} \\
& \times \frac{i}{\not p_{l T}} u^{r}(p)\left(-x_{1} \sin \theta_{W}+x_{2} \cos \theta_{W}\right), \\
& i \mathcal{M}_{Z / \gamma}^{4}=-y_{N} \bar{u}^{s}\left(\tilde{p}_{1}\right) P_{R} u^{r}(p) .
\end{aligned}
$$

The total result of the squared amplitude is

$$
\begin{aligned}
A_{Z / \gamma, t}= & \sum_{r, s=1,2} \mathcal{M}_{Z / \gamma}^{n} \mathcal{M}_{Z / \gamma}^{* m} \epsilon_{t n}^{Z / \gamma} \epsilon_{t m}^{Z / \gamma *} f_{F}\left(\frac{p_{1}^{0}}{T}\right) \\
& \times f_{B}\left(\frac{p_{2}^{0}}{T}\right) Z_{l}\left(p_{1}\right) Z_{Z / \gamma t}\left(p_{2}\right),
\end{aligned}
$$

where the "renormalization constant" $Z_{Z / \gamma(T / L \text { out })}\left(p_{2}\right)$ is calculated to be

$$
Z_{Z / \gamma(T, L)}\left(p_{2}\right)=\frac{2 p_{2}^{0}}{2 p_{2}^{0}-\Pi_{(T, L), p_{2}^{0}}^{Z / \gamma, \text { on shell }}\left(p_{2}\right)},
$$

and $\Pi_{(T, L), p_{2}^{0}}^{Z / \gamma, \text { on shell }}\left(p_{2}\right)=x^{T} \frac{\partial \Pi_{T, L}^{Z / \gamma}\left(p_{2}\right)}{\partial p_{2}^{0}} x$, so that

$\Pi_{T, L}^{Z / \gamma, \text { on shell }}\left(p_{2}\right)=x_{1}^{2} \frac{\partial \Pi_{T, L}^{B}\left(p_{2}\right)}{\partial p_{2}^{0}}+x_{2}^{2} \frac{\partial \Pi_{T, L}^{W}\left(p_{2}\right)}{\partial p_{2}^{0}}$.

\section{Goldstone channels}

Besides the Goldstone components in the $Z_{L}$ and $W_{L}$ polarization vectors, the Goldstone boson's fragments also contribute to the $1 \leftrightarrow 2$ rate. Rigorously speaking, these remains are no longer a "particle," since they are "branch cuts" rather than "poles." However, since the imaginary parts peak significantly at $p_{2}^{0}= \pm\left|\vec{p}_{2}\right|$, we can apply the approximation to regard them as massless bosons. The corresponding Feynman diagrams are the same as the second panels in Figs. 1 and 2, with the only difference being that the Goldstone boson's components are no longer bounded with the longitudinal polarizations of the $W$ and $Z$ bosons. 


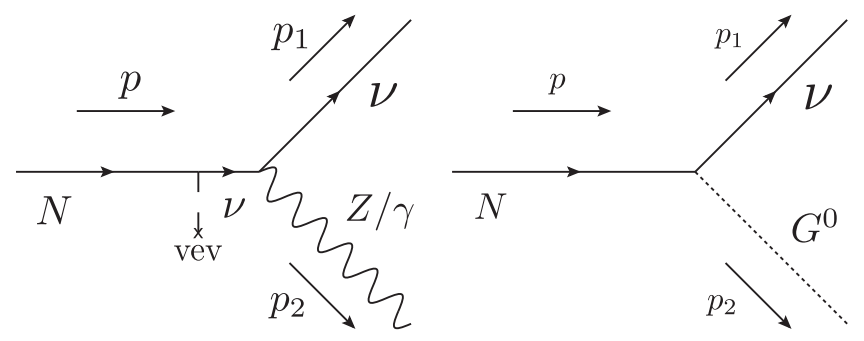

FIG. 2. $\quad N \rightarrow(Z / \gamma) \nu 1 \leftrightarrow 2$ channel.

The dispersion relation of a "massless" Goldstone boson is simple:

$$
F_{G}\left(p_{2}\right)=\left(p_{2}^{0}\right)^{2}-\vec{p}_{2}^{2}=0 .
$$

Other equations are the same as in the previous subsections. After solving Eqs. (10), (13), and (14) with Eq. (37), we then write down the final result of the squared amplitude

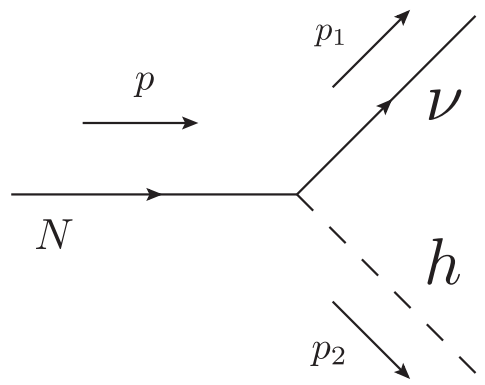

FIG. 3. $\quad N \rightarrow h \nu 1 \leftrightarrow 2$ channel.
$A_{G^{ \pm}}=\sum_{r, s=1,2} \mathcal{M}_{W}^{4} \mathcal{M}_{W}^{* 4} f_{F}\left(\frac{p_{1}^{0}}{T}\right) f_{B}\left(\frac{p_{2}^{0}}{T}\right) Z_{l}\left(p_{1}\right) Z_{G^{ \pm}}\left(p_{2}\right)$

for the charged Goldstone channel, where $Z_{G^{ \pm}}\left(p_{2}\right)$ is calculated and defined by

$$
Z_{G^{ \pm}}\left(p_{2}\right)=\frac{2\left|\vec{p}_{2}\right|}{\pi} \int_{0}^{|\vec{k}|+\delta} \operatorname{Im}\left[i \frac{p_{2}^{2}-\Pi_{L}^{W}\left(p_{2}\right)+i \epsilon}{p_{2}^{2}-\left[m_{W}(T)\right]^{2}-\Pi_{L}^{W}\left(p_{2}\right)+i \epsilon} \frac{i}{p_{2}^{2}+i \epsilon}\right] d k^{0},
$$

and the final result

$$
A_{G^{0}}=\sum_{r, s=1,2} \mathcal{M}_{Z / \gamma}^{4} \mathcal{M}_{Z / \gamma}^{* 4} f_{F}\left(\frac{p_{1}^{0}}{T}\right) f_{B}\left(\frac{p_{2}^{0}}{T}\right) Z_{l}\left(p_{1}\right) Z_{G^{0}}\left(p_{2}\right)
$$

for the neutral Goldstone channel, where

$$
Z_{G^{0}}\left(p_{2}\right)=\frac{2\left|\vec{p}_{2}\right|}{\pi} \int_{0}^{|\vec{k}|+\delta} \operatorname{Im}\left[i \frac{\left(p_{2}^{2}-\Pi_{L}^{11}+i \epsilon\right)\left(p_{2}^{2}-\Pi_{L}^{22}+i \epsilon\right)-\left(\Pi_{L}^{12}\right)^{2}}{\left(p_{2}^{2}-\left[m_{Z}(T)\right]^{2}-\Pi_{L}^{11}+i \epsilon\right)\left(p_{2}^{2}-\Pi_{L}^{22}+i \epsilon\right)-\left(\Pi_{L}^{12}\right)^{2}} \frac{i}{p_{2}^{2}+i \epsilon} .\right.
$$

Here, $m_{Z}(T)=\frac{\sqrt{g_{1}^{2}+g_{2}^{2}}}{2} v(T)$, and

$$
\begin{aligned}
& \Pi_{L}^{11}=\Pi_{L}^{B} \sin ^{2} \theta_{W}+\Pi_{L}^{W} \cos ^{2} \theta_{W}, \\
& \Pi_{L}^{22}=\Pi_{L}^{B} \cos ^{2} \theta_{W}+\Pi_{L}^{W} \sin ^{2} \theta_{W}, \\
& \Pi_{L}^{12}=\Pi_{L}^{W} \cos \theta_{W} \sin \theta_{W}-\Pi_{L}^{B} \cos \theta_{W} \sin \theta_{W} .
\end{aligned}
$$

\section{Higgs channels}

The Higgs channel, as illustrated in Fig. 3, is quite straightforward, since the Higgs boson only receives a trivial mass correction from the thermal environment. Below the $T_{c}, m_{h}(T) \propto v(T)$, so

$$
m_{h}(T)=m_{h 0} \sqrt{1-\frac{T^{2}}{T_{c}^{2}}}
$$

and above the $T_{c}, m_{h}(T)$ becomes

$$
m_{h}^{2}(T)=\left(g_{1}^{2}+3 g_{2}^{2}+4 y_{t}^{2}+8 \lambda\right) \frac{T^{2}-T_{c}^{2}}{16},
$$

where $m_{h 0}=125 \mathrm{GeV}$. Therefore, the dispersion relation of a Higgs boson is simply

$$
F_{H}\left(p_{2}\right)=p_{2}^{2}-m_{h}(T)^{2}=0 .
$$

Again solving Eqs. (10), (13), and (14) with Eq. (45) for the valid $p_{1}$ and $p_{2}$, we then write down the amplitude,

$$
i \mathcal{M}_{h}=i y_{N} \bar{u}^{s}\left(\tilde{p}_{1}\right) P_{R} u^{r}(p)
$$

The total result of the squared amplitude is

$$
A_{h}=\sum_{r, s=1,2} \mathcal{M}_{h} \mathcal{M}_{h}^{*} f_{F}\left(\frac{p_{1}^{0}}{T}\right) f_{B}\left(\frac{p_{2}^{0}}{T}\right) Z_{l}\left(p_{1}\right)
$$




\section{PHASE SPACE AND THERMAL AVERAGE INTEGRATION}

In the thermal background, the Lorentz invariance is broken, so we cannot directly "boost" the center-of-momentum reference frame to calculate the $1 \leftrightarrow 2$ processes of a sterile neutrino at rest. We can only rely on the definition of a width at an arbitrary reference frame:

$$
\begin{aligned}
\Gamma_{X} & =\frac{1}{2 p^{0}} \int \frac{d^{3} \vec{p}_{1} d^{3} \vec{p}_{2}}{(2 \pi)^{6}} \frac{A_{X}}{\left(2 p_{1}^{0}\right)\left(2 p_{2}^{0}\right)}(2 \pi)^{4} \delta^{4}\left(p-p_{1}-p_{2}\right) \\
& =\frac{1}{2 p^{0}} \int \frac{d^{3} \vec{p}_{1}}{(2 \pi)^{6}} \frac{A_{X}}{\left(2 p_{1}^{0}\right)\left(2 p_{2}^{0}\right)}(2 \pi)^{4} \delta\left(p^{0}-p_{1}^{0}-p_{2}^{0}\right) \\
& =\frac{1}{2 p^{0}} \int \frac{2 \pi \sin \theta_{p} \vec{p}_{1}^{2} d\left|\vec{p}_{1}\right| d \theta_{p}}{(2 \pi)^{6}} \frac{A_{X}}{\left(2 p_{1}^{0}\right)\left(2 p_{2}^{0}\right)}(2 \pi)^{4} \delta\left(p^{0}-p_{1}^{0}-p_{2}^{0}\right),
\end{aligned}
$$

where $\quad X=[W,(T, L$ out $)],[Z / \gamma,(T, L$ out $)], G^{ \pm}, G^{0}, h$. Note that in the thermal plasma rest frame, there is still the symmetry of the system rotating along the $\vec{p}$ axis, so the $\phi$ parameter of the spherical coordinate is eliminated and replaced with a $2 \pi$ factor. To integrate out the $\delta$ function, we calculate

$$
\frac{\partial p_{1}^{0}}{\partial\left|\vec{p}_{1}\right|}+\frac{\partial p_{2}^{0}}{\partial\left|\vec{p}_{1}\right|}=\frac{\partial p_{1}^{0}}{\partial\left|\vec{p}_{1}\right|}+\frac{\partial p_{2}^{0}}{\partial\left|\vec{p}_{2}\right|} \frac{\partial\left|\vec{p}_{2}\right|}{\partial\left|\vec{p}_{1}\right|}
$$

$\frac{\partial\left|\vec{p}_{2}\right|}{\partial\left|\vec{p}_{1}\right|}$ is extracted from the momentum conservation law [Eq. (14)], and the result is

$$
\frac{\partial\left|\vec{p}_{2}\right|}{\partial\left|\vec{p}_{1}\right|}=\frac{\left|\vec{p}_{1}\right|-|\vec{p}| \cos \theta_{p}}{\left|\vec{p}_{2}\right|}
$$

$\frac{\partial p_{1}^{0}}{\partial\left|\vec{p}_{1}\right|}$ and $\frac{\partial p_{2}^{0}}{\partial\left|\vec{p}_{2}\right|}$ can be extracted from the corresponding dispersion relations [Eqs. (5), (30), (37), and (45)]. Generally, if the dispersion relation of a momentum $p_{Y}$ is written as $F_{X}\left(p_{Y}\right)=F_{X}\left(p_{Y}^{0},\left|\vec{p}_{Y}\right|\right)=0$, where $X=l$, [W, $(T, L$ out $)],[Z / \gamma,(T$, Lout $)], G, H$, then

$$
\frac{\partial p_{Y}^{0}}{\partial\left|\vec{p}_{Y}\right|}=-\frac{\frac{\partial F_{X}\left(p_{Y}\right)}{\partial\left|\vec{p}_{Y}\right|}}{\frac{\partial F_{X}\left(p_{Y}\right)}{\partial p_{Y}^{0}}} .
$$

Therefore, Eq. (49) can be reduced to

$$
\begin{aligned}
& \Gamma_{X}=\frac{1}{2 p^{0}} \int \frac{2 \pi \sin \theta_{p} \vec{p}_{1}^{2} d \theta_{p}}{(2 \pi)^{6}} \frac{A_{X}}{\left(2 p_{1}^{0}\right)\left(2 p_{2}^{0}\right)} \\
& \times \frac{(2 \pi)^{4}}{\mid \frac{\partial F_{1}\left(p_{1}\right)}{\frac{\left.\partial \vec{p}_{1}\right)}{\partial F_{l}\left(p_{1}\right)}}+\frac{\frac{\partial F_{X}\left(p_{2}\right)}{\partial p_{1}^{0}}}{\frac{\partial \vec{p}_{2} \mid}{\partial F_{X}\left(p_{2}\right)}} \frac{\left|\vec{p}_{1}\right|-|\vec{p}| \cos \theta_{p} \mid}{\partial p_{2}^{0}}} .
\end{aligned}
$$

The thermal average integration is then simple:

$$
\gamma_{X}=\int \frac{d^{3} \vec{p}}{(2 \pi)^{3}} e^{-\frac{p^{0}}{T}} \Gamma_{X}
$$

This $\gamma_{X}$ will enter the Boltzmann equation.

Straightforwardly applying Eqs. (52) and (53) raises a problem. For each $\theta_{p}$, sometimes there are multiple solutions for the $p_{1}^{0}, \vec{p}_{1}, p_{2}^{0}, \vec{p}_{2}$ values. One reason is that when a particle decays to every direction in its center-ofmomentum frame, and while boosted to the plasmareference frame, one angle can pick up multiple different momenta. To address this problem, one can adjust the integration order to perform the calculation in the (inverse) decayed particle's rest frame.

For example, for a sterile neutrino's decay process, we rely on the $N$-rest frame by boosting $p_{1}, p_{2}$ into $p_{1 N}, p_{2 N}$. We then use $p_{1,2 N}$ as the input parameters to solve the various dispersion relations. We next need to calculate the Jacobian and delta function's factors in the new $p_{1 N}, p_{2 N}$ parameters. We take the $x$ axis along the $\vec{p}$ direction, and without loss of generality, let $\vec{p}_{1}$ be located in the $x-y$ plane, and we have

$$
\begin{gathered}
p_{1 N}^{0}=\gamma\left(p_{1}^{0}-\beta\left|\vec{p}_{1}\right| \cos \theta_{p}\right), \\
\left|\vec{p}_{1 N}\right| \cos \theta_{N}=\gamma\left(\left|\vec{p}_{1}\right| \cos \theta_{p}-\beta p_{1}^{0}\right),
\end{gathered}
$$

$$
\left|\vec{p}_{1 N}\right| \sin \theta_{N}=\left|\vec{p}_{1}\right| \sin \theta_{p}
$$

where $\beta=\frac{|\vec{p}|}{p^{0}}, \gamma=\frac{1}{\sqrt{1-\beta^{2}}}$. A tedious calculation finally shows that

$$
\frac{d \theta_{N}}{d \theta_{p}}=\frac{\partial \theta_{N}}{\partial \theta_{p}}+\frac{\partial \theta_{N}}{\partial p_{1}^{0}} \frac{\partial p_{1}^{0}}{\partial \theta_{p}}+\frac{\partial \theta_{N}}{\partial\left|\vec{p}_{1}\right|} \frac{\partial\left|\vec{p}_{1}\right|}{\partial \theta_{p}},
$$

where 
TABLE I. Channels to be plotted and their meanings.

\begin{tabular}{lccc}
\hline \hline Alias & Meaning & Alias & Meaning \\
\hline WTD & $N \leftrightarrow W_{T}^{+} l^{-}$ & Z $\gamma$ LID & $N \bar{v} \leftrightarrow Z_{L} / \gamma_{L}$ \\
WTID & $N l^{+} \leftrightarrow W_{T}^{+}$ & $G^{ \pm} \mathrm{D}$ & $N \leftrightarrow G^{+} l^{-}$ \\
WLD & $N \leftrightarrow W_{L}^{+} l^{-}$ & $G^{ \pm} \mathrm{ID}$ & $N G^{-} \leftrightarrow l^{-}, N l^{+} \leftrightarrow G^{+}$ \\
WLID & $N l^{+} \leftrightarrow W_{L}^{+}$ & $G^{0} \mathrm{D}$ & $N G^{0} \leftrightarrow \nu, N \bar{\nu} \leftrightarrow G^{0}$ \\
Z $\gamma$ TD & $N \leftrightarrow Z_{T} / \gamma_{T} \nu$ & $G^{0} \mathrm{ID}$ & $N G^{0} \leftrightarrow \nu, N \bar{\nu} \leftrightarrow G^{0}$ \\
Z $\gamma$ TID & $N \bar{\nu} \leftrightarrow Z_{T} / \gamma_{T}$ & HD & $N \leftrightarrow h \nu$ \\
Z $\gamma$ LD & $N \leftrightarrow Z_{L} / \gamma_{L} \nu$ & HID & $N h \leftrightarrow \nu, N \bar{\nu} \leftrightarrow h$ \\
\hline \hline
\end{tabular}

$\frac{\partial \theta_{N}}{\partial \theta_{p}}=\frac{\vec{p}_{1}^{2} \gamma\left(-\left|\vec{p}_{1}\right|+p_{1}^{0} \beta \cos \theta_{p}\right) \sin \theta_{p}}{\left[\gamma^{2}\left(p_{1}^{0} \beta-\left|\vec{p}_{1}\right| \cos \theta_{p}\right)^{2}+\vec{p}_{1}^{2} \sin ^{2} \theta_{p}\right]^{\frac{3}{2}}} \frac{1}{\left(-\sin \theta_{N}\right)}$,

$\frac{\partial \theta_{N}}{\partial p_{1}^{0}}=\frac{-\vec{p}_{1}^{2} \beta \gamma \sin ^{2} \theta_{p}}{\left[\gamma^{2}\left(p_{1}^{0} \beta-\left|\vec{p}_{1}\right| \cos \theta_{p}\right)^{2}+\vec{p}_{1}^{2} \sin ^{2} \theta_{p}\right]^{\frac{3}{2}}} \frac{1}{\left(-\sin \theta_{N}\right)}$,

$$
\frac{\partial \theta_{N}}{\partial\left|\vec{p}_{1}\right|}=\frac{p_{1}^{0}\left|\vec{p}_{1}\right| \beta \gamma \sin ^{2} \theta_{p}}{\left[\gamma^{2}\left(p_{1}^{0} \beta-\left|\vec{p}_{1}\right| \cos \theta_{p}\right)^{2}+\vec{p}_{1}^{2} \sin ^{2} \theta_{p}\right]^{\frac{3}{2}}} \frac{1}{\left(-\sin \theta_{N}\right)},
$$

$$
\begin{gathered}
\frac{\partial p_{1}^{0}}{\partial \theta_{p}}=\frac{|\vec{p}|\left|\vec{p}_{1}\right| \sin \theta_{p}}{-\frac{\partial\left|\vec{p}_{1}\right|}{\partial p_{1}^{0}}\left|\vec{p}_{1}\right|-\frac{\partial\left|\vec{p}_{2}\right|}{\partial p_{2}^{0}}\left|\vec{p}_{2}\right|+\frac{\partial\left|\vec{p}_{1}\right|}{\partial p_{1}^{0}}|\vec{p}| \cos \theta_{p}}, \\
\frac{\partial \vec{p}_{1}}{\partial \theta_{p}}=\frac{|\vec{p}|\left|\vec{p}_{1}\right| \sin \theta_{p}}{-\vec{p}_{1}-\frac{\partial \vec{p}_{2}}{\partial p_{2}^{0}} \frac{\partial p_{1}^{0}}{\partial\left|\vec{p}_{1}\right|}\left|\vec{p}_{2}\right|+|\vec{p}| \cos \theta_{p}},
\end{gathered}
$$

and $\frac{\partial\left|\vec{p}_{i}\right|}{\partial p_{i}^{0}}$ has been already calculated in Eq. (51). Then we can replace the $d \theta_{p}$ with $d \theta_{N} \frac{d \theta_{p}}{d \theta_{N}}$ in Eq. (52) to calculate this integral.

Inverse-decay processes are similar. For example, if we calculate the $W$ boson's inverse decay process $\mathrm{Nl}^{+} \rightarrow W^{+}$, we need to adjust the integration order of Eqs. (52) and (53) to integrate out the $d^{3} \vec{p}$ and $d^{3} \vec{p}_{1}$ phase space at first, and finally to calculate the $d^{3} \vec{p}_{2}$ integration. We boost the $W^{+}$'s rest frame to transfer to $\vec{p}_{W}, \vec{p}_{1 W}$ integration by replacing the corresponding indices in Eqs. (54)-(62) to calculate the similar Jacobian and delta function's factors. With this method, all the $1 \leftrightarrow 2$ channels can be computed.

Let us summarize the numerical algorithm processes. To calculate one channel-e.g., $N \leftrightarrow W l$-one needs to follow these steps:

(1) Fixing $p^{0}, \vec{p}$, and $\theta_{N}$, solve $p_{1 N}^{0}, p_{2 N}^{0}, \vec{p}_{1 N}$, and $\vec{p}_{2 N}$. The equations to be solved are Eqs. (5), (10), (13), and (14). They are defined with the parameters $p_{1}^{0}$, $p_{2}^{0}, \vec{p}_{1}, \vec{p}_{2}$ and $\theta_{p}$, and these two sets of parameters are mediated by Eqs. (54)-(56).

(2) With the acquired numerical solution of $p_{1}, p_{2}$, and $\theta_{p}$, calculate the total squared amplitude through Eq. (22).

(3) Changing $\theta_{N}$, utilize Eqs. (52) and (57) to compute $\Gamma_{W, T / L}$.

(4) Change $p$ to calculate Eq. (53).

To calculate, e.g., the $N l \leftrightarrow W$ channel, we first need to integrate out $\vec{p}$ and $\vec{p}_{1}$. Thus, we exchange $p$ and $p_{2}$ in the above items, and we also change the subscript $N$ to $W$. Therefore, we are also able to calculate the inverse decay rate of a $W$ boson below its threshold.

\section{NUMERICAL RESULTS}

We have scanned the $m_{N} \in[50,200] \mathrm{GeV}$ range by an interval of $1 \mathrm{GeV}$. For the leptonic sector, both the "particle" and "hole" channels have been included. For the bosonic sector, all the transverse and longitudinal vector bosons, and the Goldstone and Higgs channels have been considered. We have enumerated all the $1 \leftrightarrow 2$ possibilities; however, it is unnecessary to plot all of them. We sum over the results into 14 channels, and we show their meanings in Table I. Notice that the channel $N(W / Z) \leftrightarrow$ $l^{-} / \nu$ is kinematically forbidden in our interested parameter space, so it is neglected. Compared with the production rate $\gamma_{X}$, it is more convenient to use the averaged decay width

$$
\bar{\Gamma}_{X}=\frac{g_{N} \gamma_{X}}{n_{N}^{\mathrm{eq}}}=\frac{\int \frac{d^{3} \vec{p}}{(2 \pi)^{3}} e^{-\frac{p^{0}}{T}} \Gamma_{X}}{2 \frac{m_{N}^{2} T}{2 \pi^{2}} K_{2}\left(\frac{m_{N}}{T}\right)},
$$

where $g_{N}$ is the degree of freedom of the sterile neutrino, and it is canceled by the same factor in $n_{N}^{\mathrm{eq}}$. The comparison of this parameter with the Hubble constant $H \simeq 1.66 \sqrt{g_{*}} \frac{T^{2}}{M_{\mathrm{pl}}}$ can help us judge whether the sterile neutrino starts to deviate from the thermal equilibrium conveniently.

In Fig. 4, we have selected the masses $m_{N}=50,100$, 150 , and $200 \mathrm{GeV}$ to plot their thermal averaged widths normalized by $\frac{1}{\left|y_{N}\right|^{2}}$ depending on the temperature $T$. Just below the critical temperature $100 \mathrm{GeV} \lesssim T<T_{c}$, the longitudinal $W / Z$ and the Goldstones play crucial roles. These two kinds of channels are complementary, and they can be compared with the corresponding parts of Fig. 1 in Ref. [40], in which large areas had been kinematically forbidden within the $100 \mathrm{GeV} \lesssim T<T_{c}$ and $50 \mathrm{GeV} \lesssim$ $m_{N} \lesssim 100 \mathrm{GeV}$ ranges. Our calculations do not give such a remarkable suppression. To show this clearly, we also plot a total thermal-averaged width $\bar{\Gamma}_{\text {tot }}=\sum_{X} \bar{\Gamma}_{X}$ in Fig. 5. There we can see a similar suppression of the total thermalaveraged width when $T>T_{c}$ compared with Fig. 1 in 

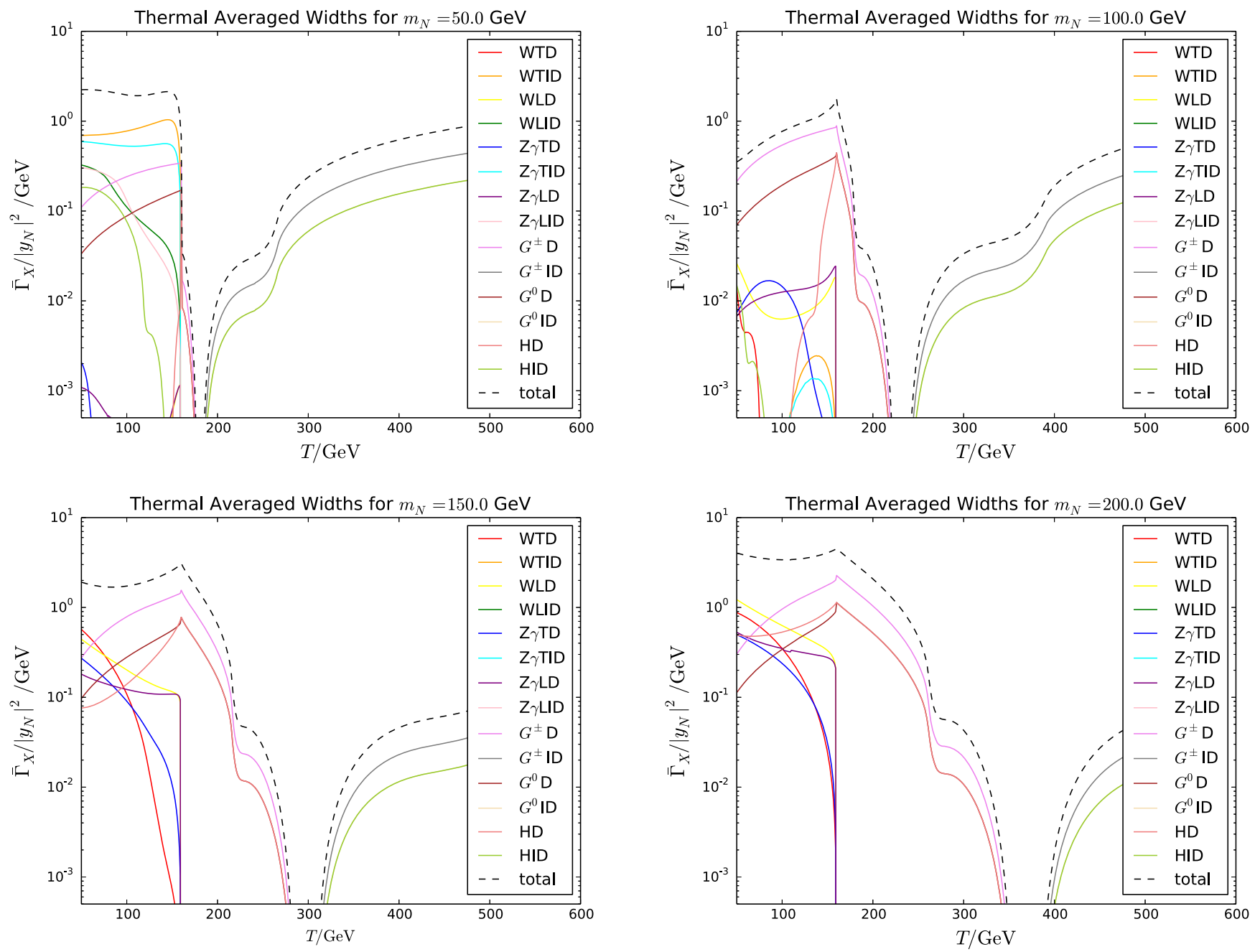

FIG. 4. Thermal averaged widths plot normalized by $\frac{1}{\left|y_{N}\right|^{2}}$ for $m_{N}=50,100,150,200 \mathrm{GeV}$ masses. The meanings in the legends are illustrated in Table I.

Ref. [40], while when $T<T_{c}$, only a slight and obscure suppression appears in roughly the same area.

In the rest of this section, we show a preliminary calculation of leptogenesis with all the results above. Above the sphaleron decoupling temperature-i.e., when $T>T_{\text {sph }}=131.7 \mathrm{GeV}[51]$ - the $B+L$ number is not conserved, so the lepton-number asymmetry generated from the sterile neutrino $1 \leftrightarrow 2$ processes is ported to the baryon-number asymmetry through the sphaleron effects. To explain the observed ratio of baryon asymmetry normalized by the photon number density $\left|\eta_{B 0}\right|=\frac{\left|n_{B}-n_{\bar{B}}\right|}{n_{\gamma}} \approx$ $6 \times 10^{-10}$ in our current Universe, $\left|\eta^{L}\right|=\frac{\left|n_{L}-n_{\bar{L}}\right|}{n_{\gamma}}$ is then calculated to be $2.47 \times 10^{-8}$ [37] at $T=T_{\text {sph }}=$ $131.7 \mathrm{GeV}$. Including the $2 \leftrightarrow 2$ washout terms, the Boltzmann equations are given by

$$
\begin{aligned}
& \frac{n_{\gamma} H_{N}}{z} \frac{d \eta_{N}}{d z}=\left(1-\frac{\eta_{N}}{\eta_{N}^{\mathrm{eq}}}\right)\left[\gamma_{D}+2\left(\gamma_{H s}+\gamma_{A s}\right)+4\left(\gamma_{H t}+\gamma_{A t}\right)\right], \\
& \frac{n_{\gamma} H_{N}}{z} \frac{d \eta_{L}}{d z}=\gamma_{D}\left[\left(\frac{\eta_{N}}{\eta_{N}^{\mathrm{eq}}}-1\right) \epsilon_{\mathrm{CP}}(z)-\frac{2}{3} \eta_{L}\right]-\frac{4}{3} \eta_{L}\left[2\left(\gamma_{H t}+\gamma_{A t}\right)+\frac{\eta^{N}}{\eta_{\mathrm{eq}}^{N}}\left(\gamma_{H s}+\gamma_{A s}\right)\right],
\end{aligned}
$$

where $\eta_{N}=\frac{n_{N}}{n_{\gamma}}, z=\frac{n_{N}}{T}$, and $\gamma_{D}=\sum_{X} \gamma_{X}$ is the summation over all the $1{ }^{\gamma} \leftrightarrow 2$ channels defined in Eq. (53). We shall neglect the $2 \leftrightarrow 2$ contributions $\gamma_{H s, H t, A s, A t}$ in this paper, since we only calculate the situation in which the sterile neutrino is initially in thermal equilibrium with the plasma when $T \gg m_{N}$. When $T \sim m_{N}$ or $T \lesssim m_{N}$, such that the 


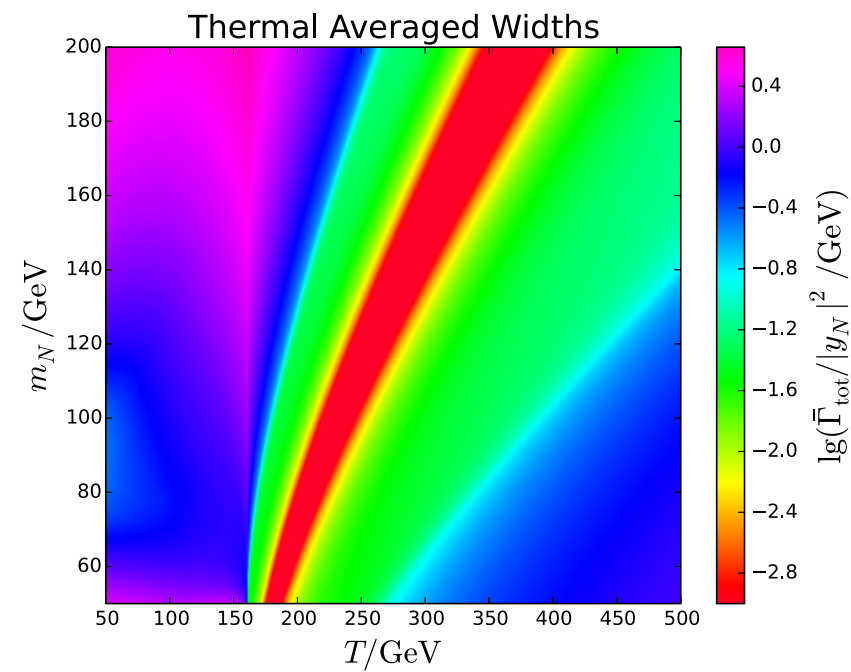

FIG. 5. $\quad \bar{\Gamma}_{\text {tot }}=\sum_{X} \bar{\Gamma}_{X}$ normalized by $\frac{1}{\left|y_{N}\right|^{2}}$, depending on the temperature $T$ and the sterile neutrino mass $m_{N}$. To keep the image contrast in the other areas where the $1 \leftrightarrow 2$ processes are not suppressed kinematically, we just keep $\frac{\bar{\Gamma}_{\text {tot }}}{\left|y_{N}\right|^{2}} \geq 1.0 \times 10^{-3} \mathrm{GeV}$ in this image. Therefore, most of the red parts in this image are actually much smaller than those plotted here.

deviation from the thermal equilibrium becomes significant, the $2 \leftrightarrow 2$ processes are usually suppressed by an additional $n_{A, H, \ldots}^{e q}$ factor compared with $\gamma_{D}$. The $C P$-source parameter $\epsilon_{\mathrm{CP}}(z)$ originates from the one-loop interference with the tree-level amplitudes [32,36] and should depend on $z$. The identification of this parameter is beyond the scope of this paper. We only follow Sec. II of Ref. [40] to regard $\epsilon_{\mathrm{CP}}$ as a constant parameter to present our results for successful leptogenesis in Fig. 6. Studies at some proposed future leptonic colliders, with the aid of secondary vertex

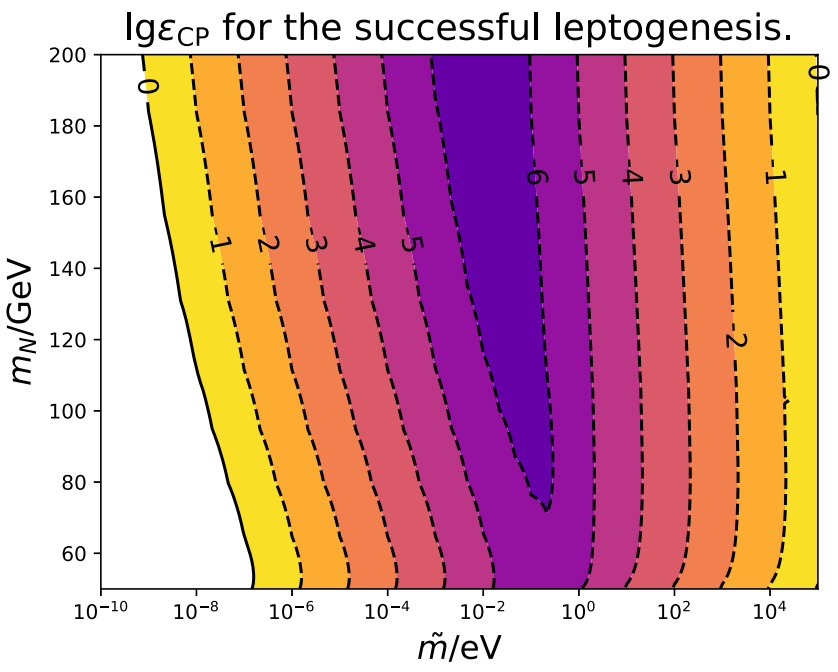

FIG. 6. $\lg \epsilon_{\mathrm{CP}}$ needed to obtain successful leptogenesis. The sterile neutrinos are initially in thermal equilibrium with the plasma. detection and the sensitivity to $y_{N}$ at ILC [52-56], CEPC [57,58], and FCC-ee [59] can be significantly improved. References [60-64] have discussed the corresponding searches at these colliders, Refs. [65,66] have also discussed the proposals at the LHeC [67,68], and Ref. [69] has discussed the similar parameter space at the LHC and beyond. Their results can roughly verify the parameter space within $50 \mathrm{GeV}<m_{N}<90 \mathrm{GeV}$ and $\tilde{m} \gtrsim 1 \mathrm{eV}$. Our contours differ significantly from Fig. 3 in Ref. [40], especially for the $1 \mathrm{eV} \lesssim \tilde{m} \lesssim 10^{5} \mathrm{eV}$ and $40 \mathrm{GeV} \lesssim$ $m_{N} \lesssim 110 \mathrm{GeV}$ area there, where quite a large void appears due to the absence of the $\gamma_{D}$ kinematically forbidden below $T_{c}$ in their Fig. 1. In our paper, this area is filled up with the $N \leftrightarrow G^{+, 0}\left(l^{-} / \nu\right), N \leftrightarrow W_{T, L}^{+} l^{-}$, or $N \leftrightarrow Z_{T, L} \nu$ channels, so that no significant distortions of the contours appear.

\section{SUMMARY}

We have calculated the $1 \leftrightarrow 2$ processes of a sterile neutrino interacting with the gauge/Higgs bosons and leptons in the thermal plasma. We applied the Goldstoneequivalence gauge to evaluate the processes below the critical temperature $T_{c} \approx 160 \mathrm{GeV}$, and our method is suitable for the sterile neutrino's mass $m_{N} \sim T_{c}$. Although a particular gauge was applied, the results are independent on gauge selections, since we have included the Eq. (A4) terms. The results can be utilized in studies involving the sterile neutrinos, and we have preliminarily calculated the leptogenesis as an example. Compared with Ref. [40], the results had been significantly changed due to the different kinematic threshold understandings in this paper. $1 \leftrightarrow 2$ results are usually sufficient to study the processes in a temperature that is roughly of the same magnitude of the sterile neutrino's mass if one assumes an initially thermal equilibrium. Yet the nonperturbative corrections, in which the leptons and bosons interchange soft particles with the plasma and with each other, have not been included. To carry forward our research to a wider temperature scale and to a more precise calculation, we will include all these effects in our further studies.

\section{ACKNOWLEDGMENTS}

We thank Junmou Chen, Pyungwon Ko, Ligong Bian, Fa-Peng Huang, Chun Liu, Chen Zhang, Ye-Ling Zhou, Mikko Laine, and Kechen Wang for helpful discussions and communications. This work is supported in part by the National Natural Science Foundation of China under Grants No. 11805288, No. 11875327, and No. 12005312; by the Natural Science Foundation of Guangdong Province under Grant No. 2016A030313313; by the Fundamental Research Funds for the Central Universities; and by the Sun Yat-Sen University Science Foundation. Part of the calculation was performed on TianHe-2, and we express thanks for the support of National Supercomputing Center in Guangzhou (NSCC-GZ). 


\section{APPENDIX: ASPECT FROM THE $\boldsymbol{R}_{\xi}$ GAUGE}

The advantage of the Goldstone equivalent gauge is the anatomy of the longitudinal polarization and the remaining Goldstone degrees of freedom contributions, which make it convenient for one to follow a "tree-level" methodology. The result should be numerically equivalent to the traditional aspect in order to calculate the imaginary part of the one-loop propagators. In fact, we showed in Ref. [45] that similar "tree-level" logic can also be applied in the standard $R_{\xi}$ gauge if only the remaining Goldstone degree of freedom is replaced by a "vector boson" with the polarization vector $\propto p$, where $p$ is the vector boson's momentum. The equivalence of the results with different gauges is guaranteed by the Ward-Takahashi identity in the broken phase [70]:

$$
p_{2}^{\mu} \mathcal{M}_{V \mu}=i m_{V}(T) \mathcal{M}^{\mathrm{GS}},
$$

where $V=Z / W, m_{V}(T)$ is the gauge boson's mass originating from the $\mathrm{VEV}$, and $\mathcal{M}^{\mathrm{GS}}$ is the amplitude with the corresponding gauge boson replaced by a Goldstone external leg. For the $W$ boson, we just notice the relationship between the polarization vectors under two gauges:

$$
\epsilon_{L, R_{\xi}}^{W}=\epsilon_{L \mathrm{in}}^{W}+\left(\begin{array}{c}
\frac{p_{2}^{\mu}}{\sqrt{p_{2}^{2}}} \\
-i \frac{m_{W}(T)}{\sqrt{p_{2}^{2}}}
\end{array}\right)
$$

where $\epsilon_{L, R_{\xi}}^{W}$ is the familiar polarization vector in the $R_{\xi}$ gauge. One immediately finds that the contribution from the difference between these two polarization vectors should always vanish according to the Ward-Takahashi identity in the broken phase.

For the mixing $Z / \gamma$ case, things are a little bit complicated. Notice that in Eq. (32), the mixing parameter's $-x_{1} \sin \theta_{W}+x_{2} \cos \theta_{W}$ factor is in the vertex term, while in Eq. (31), exactly the same thing is attributed to the polarization vector. Remember also that for a pure $\gamma$, it does not receive any mass from the VEV, so its amplitude completely disappears when dotted by the $p_{2}^{\mu}$. Factoring out the common $-x_{1} \sin \theta_{W}+x_{2} \cos \theta_{W}$ term, one finds that the contribution from the difference between the two polarization vectors,

$$
\epsilon_{L, R_{\xi}}^{Z / \gamma}-\epsilon_{L \text { in }}^{Z / \gamma}=\left(\begin{array}{c}
\frac{p_{2}^{\mu}}{\sqrt{p_{2}^{2}}} \\
-i\left(-x_{1} \sin \theta_{W}+x_{2} \cos \theta_{W}\right) \frac{m_{Z}(T)}{\sqrt{p_{2}^{2}}}
\end{array}\right),
$$

still vanishes in the amplitude, which is also guaranteed by Eq. (A1).
The above discussions only involve the longitudinal polarizations of the vector bosons. For the Goldstone channels, we have pointed out in Ref. [45] that these Goldstone external legs can be replaced by a "vector boson" with the polarization vector $\frac{p_{2}^{\mu}}{i m_{V}}$, equivalent to picking up the "quasipoles" corresponding to the $\propto p_{2}^{\mu} p_{2}^{\nu}$ terms in the $R_{\xi}$ propagator.

One might notice that the Ward-Takahashi identity is not rigorously satisfied perturbatively if one only keeps the tree-level part in Eqs. (17), (18), (32), and (33). This can be fixed if we introduce the hard thermal one-loop corrections to the gauge vertices (see p. 161 in Ref. [50]),

$$
\Gamma_{\mu}\left(p, p_{1}\right)=m_{f}^{2} \int_{\Omega_{\hat{k}}} \frac{d \Omega_{\hat{\hat{k}}}}{4 \pi} \frac{\hat{k}_{\mu} \hat{k}}{(p \cdot \hat{k})\left(p_{1} \cdot \hat{k}\right)},
$$

where $m_{f}$ is again given by Eq. (12), and $\hat{k}=(1, \hat{\vec{k}})$ and $\hat{\vec{k}} \cdot \hat{\vec{k}}=1$. The recovery of Eq. (A1) can be seen by dotting the $p_{2}=p-p_{1}$ into $\Gamma_{\mu}$ :

$$
\begin{aligned}
\left(p-p_{1}\right) \cdot \Gamma\left(p, p_{1}\right) & =m_{f}^{2} \int_{\Omega_{\hat{k}}} \frac{d \Omega_{\hat{\hat{k}}}}{4 \pi}\left[\frac{\hat{\not k}}{p_{1} \cdot \hat{k}}-\frac{\hat{\not k}}{p \cdot \hat{k}}\right] \\
& =\Sigma\left(p_{1}\right)-\Sigma(p),
\end{aligned}
$$

where $\Sigma(p)$ is the hard thermal one-loop correction on a fermionic propagator of the active neutrino or a charged lepton. These two $\Sigma$ 's will help cancel the denominators in the $\frac{i}{\not{ }_{(1)}-\Sigma\left(p_{(1)}\right)}$ propagators on both sides of the gauge vertex, thus resuming the Ward-Takahashi identity in the broken phase.

To analytically calculate Eq. (A4), we define the dimensionless $K_{\mu \nu}$ by

$\Gamma_{\mu}\left(p, p_{1}\right)=\frac{m_{f}^{2} K_{\mu \nu}(a, b) \gamma^{\nu}}{p^{0} p_{1}^{0}}=\frac{m_{f}^{2} K_{\mu \nu}\left(\alpha, \beta, \theta_{a b}\right) \gamma^{\nu}}{p^{0} p_{1}^{0}}$,

where

$$
\begin{aligned}
a & =\frac{p}{p^{0}}=\left(1, \alpha \frac{\vec{p}}{|\vec{p}|}\right), \\
b & =\frac{p_{1}}{p_{1}^{0}}=\left(1, \beta \frac{\vec{p}_{1}}{\left|\vec{p}_{1}\right|}\right), \\
\theta_{a b} & =\frac{\vec{p} \cdot \vec{p}_{1}}{|\vec{p}|\left|\vec{p}_{1}\right|} .
\end{aligned}
$$

Therefore,

$$
K_{\mu \nu}\left(\alpha, \beta, \theta_{a b}\right)=\int_{\Omega_{\hat{k}}} \frac{d \Omega_{\hat{\hat{k}}}}{4 \pi} \frac{\hat{k}_{\mu} \hat{k}_{\nu}}{(a \cdot \hat{k})(b \cdot \hat{k})} .
$$


Obviously, $K_{\mu \nu}=K_{\nu \mu}$, and $K_{\mu}^{\mu}=0$. It is then convenient to decompose $K_{\mu \nu}$ into a combination of the tensor basis:

$$
K_{\mu \nu}\left(\alpha, \beta, \theta_{a b}\right)=A t_{\mu} t_{\nu}+B\left(t_{\mu} a_{\nu}+t_{\nu} a_{\mu}\right)+C\left(t_{\mu} b_{\nu}+t_{\nu} b_{\mu}\right)+D a_{\mu} a_{\nu}+E b_{\mu} b_{\nu}+F\left(a_{\mu} b_{\nu}+a_{\nu} b_{\mu}\right)+G l_{\mu} l_{\nu}
$$

Here $t=(1,0,0,0)$ is the reference frame vector of the plasma, and $l=\left(0, \frac{\vec{p} \times \vec{p}_{1}}{|\vec{p}|\left|\vec{p}_{1}\right|}\right)$, which is the unit vector perpendicular to the two input momenta. One might consider extra bases such as $t_{\mu} l_{\nu}, a_{\mu} l_{\nu}$, etc. However, if we rotate to the frame in which $l=(0,1,0,0), a=(1,0,0, \alpha), b=\left(1,0, \beta \sin \theta_{a b}, \beta \cos \theta_{a b}\right)$, we find that the $t_{\mu} l_{\nu}$, etc., factors all contain integrals like $\int_{\Omega_{\hat{k}}} \frac{d \Omega_{\hat{k}}}{4 \pi} \frac{\hat{k}_{1} \hat{k}_{0,2,3}}{(a \cdot \hat{k})(b \cdot \hat{k})}$, with an integrand which is odd under the transformation $\hat{k}_{1} \rightarrow-\hat{k}_{1}$. Therefore, all these terms vanish.

We then contract the $K_{\mu \nu}$ with $t_{\mu} t_{\nu}, t_{\mu} a_{\nu}, t_{\mu} b_{\nu}, a_{\mu} a_{\nu}, b_{\mu} b_{\nu}, a_{\mu} b_{\nu}$, and $l_{\nu} l_{\nu}$ to determine the $A-G$ coefficients. Together with the traceless condition $K_{\mu}^{\mu}=0$, the expressions are

$$
\begin{aligned}
& K_{t t}=K_{\mu \nu} t^{\mu} t^{\nu}=\int_{\Omega_{\hat{\vec{k}}}} \frac{d \Omega_{\hat{\vec{k}}}}{4 \pi} \frac{1}{(a \cdot \hat{k})(b \cdot \hat{k})}=A+2 B+2 C+D+E+2 F, \\
& K_{t a}=K_{\mu \nu} t^{\mu} a^{\nu}=\int_{\Omega_{\hat{k}}} \frac{d \Omega_{\hat{\vec{k}}}}{4 \pi} \frac{1}{b \cdot \hat{k}}=A+\left(2-\alpha^{2}\right) B+\left(2-\alpha \beta \cos \theta_{a b}\right) C \\
& +\left(1-\alpha^{2}\right) D+\left(1-\alpha \beta \cos \theta_{a b}\right) E+\left(2-\alpha^{2}-\alpha \beta \cos \theta_{a b}\right) F, \\
& K_{t b}=K_{\mu \nu} t^{\mu} b^{\nu}=\int_{\Omega_{\hat{\vec{k}}}} \frac{d \Omega_{\hat{\vec{k}}}}{4 \pi} \frac{1}{a \cdot \hat{k}}=A+\left(2-\alpha \beta \cos \theta_{a b}\right) B+\left(2-\beta^{2}\right) C \\
& +\left(1-\alpha \beta \cos \theta_{a b}\right) D+\left(1-\beta^{2}\right) E+\left(2-\beta^{2}-\alpha \beta \cos \theta_{a b}\right) F, \\
& K_{a a}=K_{\mu \nu} a^{\mu} a^{\nu}=\int_{\Omega_{\hat{\vec{k}}}} \frac{d \Omega_{\hat{\vec{k}}}}{4 \pi} \frac{a \cdot \hat{k}}{b \cdot \hat{k}}=A+2\left(1-\alpha^{2}\right) B+2\left(1-\alpha \beta \cos \theta_{a b}\right) C \\
& +\left(1-\alpha^{2}\right)^{2} D+\left(1-\alpha \beta \cos \theta_{a b}\right)^{2} E+2\left(1-\alpha^{2}\right)\left(1-\alpha \beta \cos \theta_{a b}\right) F, \\
& K_{b b}=K_{\mu \nu} b^{\mu} b^{\nu}=\int_{\Omega_{\hat{k}}} \frac{d \Omega_{\hat{\vec{k}}}}{4 \pi} \frac{b \cdot \hat{k}}{a \cdot \hat{k}}=A+2\left(1-\alpha \beta \cos \theta_{a b}\right) B+2\left(1-\beta^{2}\right) C \\
& +\left(1-\alpha \beta \cos \theta_{a b}\right)^{2} D+\left(1-\beta^{2}\right)^{2} E+2\left(1-\beta^{2}\right)\left(1-\alpha \beta \cos \theta_{a b}\right) F \\
& K_{a b}=K_{\mu \nu} a^{\mu} b^{\nu}=\int_{\Omega_{\vec{k}}} d \Omega_{\hat{\vec{k}}}=A+\left(2-\alpha^{2}-\alpha \beta \cos \theta_{a b}\right) B+\left(2-\beta^{2}-\alpha \beta \cos \theta_{a b}\right) C \\
& +\left(1-\alpha^{2}\right)\left(1-\alpha \beta \cos \theta_{a b}\right) D+\left(1-\beta^{2}\right)\left(1-\alpha \beta \cos \theta_{a b}\right) E \\
& +\left[\left(1-\alpha^{2}\right)\left(1-\beta^{2}\right)+\left(1-\alpha \beta \cos \theta_{a b}\right)^{2}\right] F \text {, } \\
& K_{l l}=K_{\mu \nu} l^{\mu} l^{\nu}=\int_{\Omega_{\hat{k}}} \frac{d \Omega_{\hat{\vec{k}}}}{4 \pi} \frac{(l \cdot \hat{k})(l \cdot \hat{k})}{(a \cdot \hat{k})(b \cdot \hat{k})}=G . \\
& K_{\mu}^{\mu}=A+2 B+2 C+\left(1-\alpha^{2}\right) D+\left(1-\beta^{2}\right) E+\left(2-2 \alpha \beta \cos \theta_{a b}\right) F-G=0 .
\end{aligned}
$$

It is convenient to calculate all the integrals in Eq. (A10) within the $l=(0,1,0,0), a=(1,0,0, a), b=$ $\left(1,0, b \sin \theta_{a b}, b \cos \theta_{a b}\right)$ framework. We list the results below: 


$$
\begin{aligned}
K_{t t}= & \frac{\operatorname{artanh}\left[\frac{2\left(1-\alpha \beta \cos \theta_{a b}\right) \sqrt{\alpha^{2}+\beta^{2}-\alpha^{2} \beta^{2}+\alpha \beta \cos \theta_{a b}\left(\alpha \beta \cos \theta_{a b}-2\right)}}{1+\alpha^{2}+\beta^{2}-4 \alpha \beta \cos \theta_{a b}+\alpha^{2} \beta^{2} \cos 2 \theta_{a b}}\right]}{2 \sqrt{\alpha^{2}+\beta^{2}-\alpha^{2} \beta^{2}+\alpha \beta \cos \theta_{a b}\left(\alpha \beta \cos \theta_{a b}-2\right)}}, \\
K_{t a}= & \frac{\operatorname{artanh} \beta}{\beta}, \quad K_{t b}=\frac{\operatorname{artanh} \alpha}{\alpha}, \\
K_{a a}= & \frac{\left(\beta-\alpha \cos \theta_{a b}\right) \operatorname{artanh} \beta+\alpha \beta \cos \theta_{a b}}{\beta^{2}}, \\
K_{b b}= & \frac{\left(\alpha-\beta \cos \theta_{a b}\right) \operatorname{artanh} \alpha+\alpha \beta \cos \theta_{a b}}{\alpha^{2}}, \\
K_{a b}= & 1, \\
K_{l l}= & \frac{\operatorname{artanh} \beta\left(\beta-\alpha \cos \theta_{a b}\right)+\operatorname{artanh} \alpha\left(\alpha-\beta \cos \theta_{a b}\right)}{\alpha^{2} \beta^{2} \sin ^{2} \theta_{a b}} \\
& +\operatorname{artanh}\left[\frac{\left(\alpha \beta \cos \theta_{a b}-1\right) \sqrt{4 \alpha^{2}+4 \beta^{2}-2 \alpha^{2} \beta^{2}+2 \alpha \beta\left(\alpha \beta \cos 2 \theta_{a b}-4 \cos \theta_{a b}\right)}}{1+\alpha^{2}+\beta^{2}+\alpha \beta\left(\alpha \beta \cos 2 \theta_{a b}-4 \cos \theta_{a b}\right)}\right. \\
& \times \frac{\sqrt{4 \alpha^{2}+4 \beta^{2}-2 \alpha^{2} \beta^{2}+2 \alpha \beta\left(\alpha \beta \cos 2 \theta_{a b}-4 \cos \theta_{a b}\right)}}{4 \alpha^{2} \beta^{2} \sin ^{2} \theta_{a b}} .
\end{aligned}
$$

By plugging Eq. (A11) into Eq. (A10), we acquire eight equations with seven unknown parameters. We can solve seven of them to acquire the values $A-G$, and then $K_{\mu \nu}\left(\alpha, \beta, \theta_{a b}\right)$ is determined through Eq. (A9).

When, however, $\vec{p}$ and $\vec{p}_{1}$ are nearly parallel to each other, or when one of them is extremely small, the above method suffers from instability due to the near degeneration of the matrix corresponding to the linear equations in Eq. (A10). To cure this problem, when $\vec{p}$ and $\vec{p}_{1}$ are nearly parallel to each other, we estimate $K_{\mu \nu}$ by taking the $\theta_{a b} \rightarrow 0$ limit,

$$
\begin{aligned}
& K_{t t, \theta_{a b} \rightarrow 0}=\frac{\operatorname{artanh} \alpha-\operatorname{artanh} \beta}{\alpha-\beta}, \\
& K_{l l, \theta_{a b} \rightarrow 0}=\frac{\left(\alpha^{2}-1\right) \beta^{2} \operatorname{artanh} \alpha-\alpha^{2}\left(\beta^{2}-1\right) \operatorname{artanh} \beta}{2 \alpha^{2} \beta^{2}(\alpha-\beta)}-\frac{1}{2 \alpha \beta},
\end{aligned}
$$

or by taking the $\theta_{a b} \rightarrow \pi$ limit,

$$
\begin{aligned}
K_{t t, \theta_{a b} \rightarrow \pi} & =\frac{\operatorname{artanh} \alpha+\operatorname{artanh} \beta}{\alpha+\beta}, \\
K_{l l, \theta_{a b} \rightarrow \pi} & =\frac{\left(\alpha^{2}-1\right) \beta^{2} \operatorname{artanh} \alpha+\alpha^{2}\left(\beta^{2}-1\right) \operatorname{artanh} \beta}{2 \alpha^{2} \beta^{2}(\alpha+\beta)}+\frac{1}{2 \alpha \beta} .
\end{aligned}
$$

Then $K_{\mu \nu}$ can be expressed as

$$
\begin{aligned}
K_{\mu \nu, \vec{p} \| \vec{p}_{1}}\left(\alpha, \beta, \theta_{a b}\right)= & K_{t t} t^{\mu} t^{\nu}+K_{l l} l_{1 \mu} l_{1 \nu}+K_{l l} l_{2 \mu} l_{2 \nu} \\
& +K_{\perp}(a-t)_{\mu}(a-t)_{\nu} \\
& +\frac{K_{t t}-K_{t a}}{\alpha^{2}}\left[(a-t)_{\mu} t_{\nu}+(a-t)_{\nu} t_{\mu}\right],
\end{aligned}
$$

where $l_{1}$ and $l_{2}$ are two unit vectors perpendicular to $\vec{p}$ without the time component, and also $l_{1} \perp l_{2} . K_{\perp}=2 K_{l l}-$ $K_{t t}$ due to the traceless condition. When, in the other case, and without loss of generality, $\alpha>\beta$ and $\beta \ll 1$, we can estimate $K_{\mu \nu}$ by taking the $\beta \rightarrow 0$ limit to acquire

$$
\begin{aligned}
& K_{t t, \beta \rightarrow 0}=\frac{\operatorname{artanh} \alpha}{\alpha}, \\
& K_{l l, \beta \rightarrow 0}=\frac{\alpha+\left(\alpha^{2}-1\right) \operatorname{artanh}(\alpha)}{2 \alpha^{3}},
\end{aligned}
$$

and again

$$
\begin{aligned}
K_{\mu \nu, \beta \rightarrow 0}\left(\alpha, \beta, \theta_{a b}\right)= & K_{t t} t^{\mu} t^{\nu}+K_{l l} l_{1 \mu} l_{1 \nu}+K_{l l} l_{2 \mu} l_{2 \nu} \\
& +K_{\perp}(a-t)_{\mu}(a-t)_{\nu} \\
& +\frac{K_{t t}-K_{t a}}{\alpha^{2}}\left[(a-t)_{\mu} t_{\nu}+(a-t)_{\nu} t_{\mu}\right] .
\end{aligned}
$$

If one wants a gauge-invariant result whenever the HTL corrected dispersion relations are considered, Eq. (A4) should be included. We can estimate its contributions through a power-counting consideration. Neglecting Eq. (A4) will introduce a relative error of $\sim \frac{m_{f}^{2}}{m_{N}^{2}}$ in the final results. 
$\frac{m_{f}^{2}}{m_{N}^{2}} \lesssim 1$ induces $m_{N} \lesssim 0.15 T$, since the $W / Z / \gamma$ channels open up at $T<T_{c}$, and $0.15 T_{c}=24 \mathrm{GeV}$. Therefore, our interested range, $m_{N}>50 \mathrm{GeV}$, is sufficiently safe if we neglect the vertex thermal correction terms.

The above discussions depend on the assumption that $K_{\mu \nu} \sim 1$. However, the artanh functions in Eq. (A10) diverge when $\alpha, \beta \rightarrow 1$. This can be realized by observing the denominator of Eq. (A8), which can be close to zero when $\alpha$ and $\beta$ approach 1 . Fortunately, this usually happens when a largely boosted "hole" is created. The divergence is significantly suppressed by the "renormaliztion factor" $Z_{l}\left(p_{1}\right)=\frac{\left(p_{1}^{0}\right)^{2}-\vec{p}_{1}^{2}}{2 m_{f}^{2}} \propto e^{\frac{-\vec{p}^{2}}{m_{f}^{2}}}$ in Eq. (26). Therefore, the final integrated rate nearly remains intact, although in this paper we still reckon according to the Eq. (A4) terms.

In fact, our practical evaluation shows that the simpler tree-level vertex method gives a final result not much different from the gauge independent data shown in this paper. The Goldstone equivalence gauge also displays another advantage in the tree-level vertex approximation: If we fix on the $R_{\xi}$ gauge, it might introduce a discontinuity of the total effective decay rate over the crossover temperature $T_{c}$ up to tree level. Notice that below $T_{c}$, the Goldstone boson fraction's contributions are collected within the $p_{2}^{\mu} p_{2}^{\nu}$ terms in the gauge boson components, while when $T>T_{c}$, all the Goldstone contributions originate from the Yukawa couplings. A continuous transition between these two coupling formalisms requires Eq. (A4), and neglecting this will introduce a discontinuity. Therefore, we can see that attributing all of the "Goldstone contribution" of a vector boson to the Goldstone Yukawa couplings, just as we did in the Goldstone equivalence gauge, will automatically include the key part of the [Eq. (A4)] corrections to connect the two parts. Therefore, compared with the $R_{\xi}$ gauge, the Goldstone equivalence gauge includes more hard thermal loop corrections on vertices up to a tree-level evaluation.
[1] M. Fukugita and T. Yanagida, Phys. Lett. B 174, 45 (1986).

[2] M. Luty, Phys. Rev. D 45, 455 (1992).

[3] T. Gherghetta and G. Jungman, Phys. Rev. D 48, 1546 (1993).

[4] M. Plumacher, Z. Phys. C 74, 549 (1997).

[5] M. Plumacher, Nucl. Phys. B530, 207 (1998).

[6] W. Buchmuller and M. Plumacher, Phys. Lett. B 389, 73 (1996).

[7] W. Buchmuller and M. Plumacher, Phys. Rep. 320, 329 (1999).

[8] W. Buchmuller and M. Plumacher, Int. J. Mod. Phys. A 15, 5047 (2000).

[9] W. Buchmuller, P. Di Bari, and M. Plumacher, Ann. Phys. (Amsterdam) 315, 305 (2005).

[10] S. Davidson, E. Nardi, and Y. Nir, Phys. Rep. 466, 105 (2008).

[11] Y.-L. Tang and S.-h. Zhu, J. High Energy Phys. 03 (2016) 043.

[12] Y.-L. Tang and S.-h. Zhu, J. High Energy Phys. 01 (2017) 025 .

[13] B. Batell, T. Han, and B. Shams Es Haghi, Phys. Rev. D 97, 095020 (2018).

[14] B. Batell, T. Han, D. McKeen, and B. S. E. Haghi, Phys. Rev. D 97, 075016 (2018).

[15] M. Escudero, N. Rius, and V. Sanz, Eur. Phys. J. C 77, 397 (2017).

[16] R. Allahverdi, Y. Gao, B. Knockel, and S. Shalgar, Phys. Rev. D 95, 075001 (2017).

[17] P. Bandyopadhyay, E. J. Chun, R. Mandal, and F. S. Queiroz, Phys. Lett. B 788, 530 (2019).

[18] P. Bandyopadhyay, E. J. Chun, and R. Mandal, J. Cosmol. Astropart. Phys. 08 (2020) 019.
[19] G. Bertone, D. Hooper, and J. Silk, Phys. Rep. 405, 279 (2005).

[20] L. Bian and Y.-L. Tang, J. High Energy Phys. 12 (2018) 006.

[21] L. J. Hall, K. Jedamzik, J. March-Russell, and S. M. West, J. High Energy Phys. 03 (2010) 080.

[22] K. Kajantie, M. Laine, K. Rummukainen, and M. E. Shaposhnikov, Nucl. Phys. B466, 189 (1996).

[23] G. Giudice, A. Notari, M. Raidal, A. Riotto, and A. Strumia, Nucl. Phys. B685, 89 (2004).

[24] A. Salvio, P. Lodone, and A. Strumia, J. High Energy Phys. 08 (2011) 116.

[25] M. Laine and Y. Schroder, J. High Energy Phys. 02 (2012) 068.

[26] S. Biondini, N. Brambilla, M. A. Escobedo, and A. Vairo, J. High Energy Phys. 12 (2013) 028.

[27] B. Garbrecht, F. Glowna, and M. Herranen, J. High Energy Phys. 04 (2013) 099.

[28] M. Laine, J. High Energy Phys. 08 (2013) 138.

[29] A. Anisimov, D. Besak, and D. Bodeker, J. Cosmol. Astropart. Phys. 03 (2011) 042.

[30] D. Besak and D. Bodeker, J. Cosmol. Astropart. Phys. 03 (2012) 029.

[31] I. Ghisoiu and M. Laine, J. Cosmol. Astropart. Phys. 12 (2014) 032.

[32] A. Pilaftsis, Phys. Rev. D 56, 5431 (1997).

[33] A. Pilaftsis and T. E. Underwood, Nucl. Phys. B692, 303 (2004).

[34] M. Flanz, E. A. Paschos, and U. Sarkar, Phys. Lett. B 345, 248 (1995); 384, 487(E) (1996); 382, 447(E) (1996).

[35] L. Covi, E. Roulet, and F. Vissani, Phys. Lett. B 384, 169 (1996). 
[36] T. Frossard, M. Garny, A. Hohenegger, A. Kartavtsev, and D. Mitrouskas, Phys. Rev. D 87, 085009 (2013).

[37] P. Bhupal Dev, P. Millington, A. Pilaftsis, and D. Teresi, Nucl. Phys. B886, 569 (2014).

[38] P. S. B. Dev, P. Millington, A. Pilaftsis, and D. Teresi, Nucl. Phys. B891, 128 (2015).

[39] L. Lello, D. Boyanovsky, and R. D. Pisarski, Phys. Rev. D 95, 043524 (2017).

[40] T. Hambye and D. Teresi, Phys. Rev. Lett. 117, 091801 (2016).

[41] J. Ghiglieri and M. Laine, J. Cosmol. Astropart. Phys. 07 (2016) 015.

[42] J. Ghiglieri and M. Laine, J. High Energy Phys. 05 (2017) 132.

[43] J. Ghiglieri and M. Laine, J. High Energy Phys. 02 (2019) 014.

[44] G. Jackson and M. Laine, Nucl. Phys. B950, 114870 (2020).

[45] Y.-L. Tang, J. High Energy Phys. 10 (2020) 180.

[46] A. Anisimov, D. Besak, and D. Bodeker, J. Cosmol. Astropart. Phys. 03 (2011) 042.

[47] P. Aurenche, F. Gelis, and H. Zaraket, J. High Energy Phys. 05 (2002) 043.

[48] S. Caron-Huot, Phys. Rev. D 79, 065039 (2009).

[49] J. Chen, T. Han, and B. Tweedie, J. High Energy Phys. 11 (2017) 093.

[50] M. L. Bellac, Thermal Field Theory, Cambridge Monographs on Mathematical Physics (Cambridge University Press, Cambridge, England, 2011), ISBN 9780511885068, 9780521654777, http://www.cambridge.org/mw/academic/ subjects/physics/theoretical-physics-and-mathematicalphysics/thermal-field-theory?format=AR.

[51] M. D’Onofrio, K. Rummukainen, and A. Tranberg, Phys. Rev. Lett. 113, 141602 (2014).

[52] The International Linear Collider Technical Design Report_Volume 1: Executive Summary (2013), arXiv:1306 .6327 .
[53] The International Linear Collider Technical Design Report-Volume 2: Physics (2013), arXiv:1306.6352.

[54] The International Linear Collider Technical Design Report-Volume 3.I: Accelerator in the Technical Design Phase (2013), arXiv:1306.6353.

[55] The International Linear Collider Technical Design Report-Volume 3.II: Accelerator Baseline Design (2013), arXiv:1306.6328.

[56] H. Abramowicz et al., in The International Linear Collider Technical Design Report-Volume 4: Detectors (2013), arXiv:1306.6329.

[57] CEPC Study Group, arXiv:1809.00285.

[58] CEPC Study Group, arXiv:1811.10545.

[59] A. Abada et al. (FCC Collaboration), Eur. Phys. J. Special Topics 228, 261 (2019).

[60] F. F. Deppisch, P. Bhupal Dev, and A. Pilaftsis, New J. Phys. 17, 075019 (2015).

[61] S. Antusch, E. Cazzato, and O. Fischer, J. High Energy Phys. 12 (2016) 007.

[62] S. Antusch, E. Cazzato, and O. Fischer, Int. J. Mod. Phys. A 32, 1750078 (2017).

[63] P. Hernández, J. Jones-Pérez, and O. Suarez-Navarro, Eur. Phys. J. C 79, 220 (2019).

[64] Z.S. Wang and K. Wang, Phys. Rev. D 101, 075046 (2020).

[65] S. Mondal and S. K. Rai, Phys. Rev. D 94, 033008 (2016).

[66] A. Das, S. Jana, S. Mandal, and S. Nandi, Phys. Rev. D 99, 055030 (2019).

[67] J. A. Fernandez et al. (LHeC Study Group), J. Phys. G 39, 075001 (2012).

[68] O. Bruening and M. Klein, Mod. Phys. Lett. A 28, 1330011 (2013).

[69] A. Das, Adv. High Energy Phys. 2018, 9785318 (2018).

[70] M. S. Chanowitz and M. K. Gaillard, Nucl. Phys. B261, 379 (1985). 